\title{
The C star outer disk population of M 31 seen with the SLOAN filters ${ }^{\star} \star \star$
}

\author{
S. Demers ${ }^{1}$ and P. Battinelli ${ }^{2}$ \\ ${ }^{1}$ Département de Physique, Université de Montréal, CP 6128, Succursale Centre-Ville, Montréal, Québec H3C 3J7, Canada \\ e-mail: demers@astro.umontreal.ca \\ 2 INAF - Osservatorio Astronomico di Roma, Viale del Parco Mellini 84, 00136 Roma, Italia \\ e-mail: battinel@oarhp1.rm.astro.it
}

Received 14 December 2004 / Accepted 17 February 2005

\begin{abstract}
We employ the CFHT Megacam camera to survey $\sim$ one deg $^{2}$ of the southern outer disc of M 31 , a region which includes the area where Battinelli et al. (2003, AJ, 125, 1298) have identified nearly one thousand C stars. In the outer M 31 region not previously surveyed, we identify 361 new C star candidates, having similar photometric properties to the known ones, and confirm the slight decrease in the luminosity of $\mathrm{C}$ stars with galactocentric distances. We show that the Sloan $g^{\prime}, r^{\prime}$, $i^{\prime}$ filters are a viable approach, comparable to $(\mathrm{CN}-\mathrm{TiO})$, to identify $\mathrm{C}$ stars. We find that the $\left(g^{\prime}-r^{\prime}\right)$ colours of cool $\mathrm{C}$ stars can be so red that prohibitively long $g^{\prime}$ exposures are needed to acquire faint extragalactic C stars. This makes the Sloan filters a less promising approach to extend a $\mathrm{C}$ star survey to several Mpc. Our uniform large field survey detects the edge of M 31 disk at $\sim 35 \mathrm{kpc}$. The intermediate-age population, represented by $\mathrm{C}$ stars, extends further to $\sim 40 \mathrm{kpc}$.
\end{abstract}

Key words. galaxies: individual: M31- stars: carbon

\section{Introduction}

Photometric identification of intermediate-age carbon stars in nearby galaxies has recently been done using a combination of broad and narrow-band photometry. The $(\mathrm{CN}-\mathrm{TiO})$ technique has been applied by, for example, Battinelli et al. (2003), Nowotny et al. (2003) and Harbeck et al. (2004) while the near infrared approach has been adopted by, for example, Demers et al. (2002) and Cioni \& Habing (2005). The SLOAN Digital Sky Survey (SDSS) photometric system, described by Fukugita et al. (1996), has been used to identify carbon stars in the Galactic halo. Indeed, Krisciunas et al. (1998) were first to show that carbon stars can be differentiated from $\mathrm{M}$ stars in the $\left(r^{\prime}-i^{\prime}\right)-\left(g^{\prime}-r^{\prime}\right)$ diagram. Faint halo carbon stars were discovered, using this technique, from the SDSS database by Margon et al. (2002). The first catalogue of these halo C stars has recently been published by Downes et al. (2004) where 251 stars were identified in a $3000 \mathrm{deg}^{2}$ area. The authors evaluate that over $50 \%$ of the sample constitute nearby dwarf

^ Based on observations obtained with MegaPrime/MegaCam, a joint project of CFHT and CEA/DAPNIA, at the Canada-FranceHawaii Telescope (CFHT), which is operated by the National Research Council (NRC) of Canada, the Institut National des Sciences de l'Univers of the Centre National de la Recherche Scientifique (CNRS) of France, and the University of Hawaii.

$\star \star$ Full Table 2 is only available in electronic form at the CDS via anonymous ftp to cdsarc.u-strasbg.fr $(130.79 .128 .5)$ or via http://cdsweb.u-strasbg.fr/cgi-bin/qcat?]/A+A/436/91
C stars. Obviously only a global survey like the SDSS can tackle the Galactic halo. Surveys of nearby galaxies can, however, be done with a more conventional approach.

Battinelli et al. (2003) have identified nearly one thousand C stars in the southern outer disk of M31. Approximately $0.6 \mathrm{deg}^{2}$ were surveyed using the $\mathrm{CFH} 12 \mathrm{~K}$ mosaic. $\mathrm{C}$ stars are identified from their position on the $(\mathrm{CN}-\mathrm{TiO})$ vs. $(R-I)$ colour-colour diagram. The reliability of this technique is quite secure, as it was demonstrated by Brewer et al. (1995) and Albert et al. (2000). The M 31 sample offers the opportunity to compare the $(\mathrm{CN}-\mathrm{TiO})$ technique with the SDSS colour-colour approach. We therefore describe here our new observations which include part of the fields already observed by us and extend the M 31 disk survey to slightly larger radii.

\section{Observations and data reduction}

The observations presented in this paper consist of one Megacam field, centered on $\alpha=00: 37: 02.3, \delta=+39: 40: 50$ (J2000.0), obtained in Service Queue Observing mode in August 2003. The Megacam camera is installed at the prime focus of the $3.66 \mathrm{~m}$ Canada-France-Hawaii Telescope. The camera consists of a mosaic of $362048 \times 4612$ pixels CCDs providing a field of view of nearly one $\mathrm{deg}^{2}$, with a resolution of 0.187 arcsecond per pixel. Images were obtained through $g^{\prime}$, $r^{\prime}$ and $i^{\prime}$ SDSS filters. The observations were secured under non photometric and partly cloudy conditions. For this reason, three short exposures were taken under excellent conditions and have 
Table 1. Summary of the observations.

\begin{tabular}{lllcc}
\hline \hline Date & Filter & Exposure & Seeing $\left({ }^{\prime \prime}\right)$ & Airmass \\
\hline $2003 / 08 / 24$ & $r^{\prime}$ & $430 \mathrm{~s}$ & 1.34 & 1.12 \\
$2003 / 08 / 24$ & $r^{\prime}$ & $430 \mathrm{~s}$ & 0.93 & 1.12 \\
$2003 / 08 / 24$ & $i^{\prime}$ & $200 \mathrm{~s}$ & 0.78 & 1.12 \\
$2003 / 08 / 24$ & $g^{\prime}$ & $550 \mathrm{~s}$ & 0.97 & 1.11 \\
$2003 / 08 / 24$ & $g^{\prime}$ & $550 \mathrm{~s}$ & 0.91 & 1.12 \\
$2003 / 08 / 24$ & $g^{\prime}$ & $550 \mathrm{~s}$ & 0.91 & 1.11 \\
$2003 / 08 / 24$ & $r^{\prime}$ & $430 \mathrm{~s}$ & 0.78 & 1.09 \\
$2003 / 08 / 24$ & $g^{\prime}$ & $550 \mathrm{~s}$ & 0.95 & 1.08 \\
$2003 / 08 / 24$ & $g^{\prime}$ & $550 \mathrm{~s}$ & 0.90 & 1.07 \\
& & & & \\
$2003 / 09 / 18$ & $r^{\prime}$ & $43 \mathrm{~s}$ & 0.84 & 1.06 \\
$2003 / 09 / 18$ & $i^{\prime}$ & $20 \mathrm{~s}$ & 0.84 & 1.06 \\
$2003 / 09 / 18$ & $g^{\prime}$ & $55 \mathrm{~s}$ & 0.94 & 1.06 \\
\hline
\end{tabular}

been used to calibrate the long exposures. It turns out, however, that our adopted exposure times are somewhat too short to fully survey the very red stars of M31. Table 1 presents the journal of the observations. The $g^{\prime}, r^{\prime}$ and $i^{\prime}$ magnitudes, are calibrated with bright first generation SDSS standards (Smith et al. 2002), as explained in the CFHT/Megacam website.

The data distributed by the CFHT have been detrended. This means that the images have already been corrected with the master darks, biases, and flats. This pre-analysis produces 36 CCD images, of a given mosaic, with the same zero point and magnitude scale. The photometric reductions were done by Terapix, the data reduction center dedicated to the processing of extremely large data flow. The Terapix team, located at the Institut d'Astrophysique de Paris, matches and stacks all images taken with the same filter and, using SExtractor (Bertin \& Arnouts 1996), provides magnitude calibrated catalogues of objects in each of the combined images. SExtrator classifies objects into star or galaxy but the classification scheme breaks down for faint magnitudes. It is essentially useless for the M 31 stars. A flag is attached to each object, flag $=0$ corresponds to isolated object not affected by neighbours. As can be seen from Fig. 1, the northern part of the Megacam field is closer to the center of M31. For this reason a substantial stellar density gradient is observed across the field thus numerous stars with flag $\neq 0$ are present in the northern half. Since the astrometric calibration of the images has been done by the CFHT Service Observing team, we have equatorial coordinates as well as calibrated colours and magnitude for each object in the field.

\section{Results}

\subsection{The data}

Each calibrated $i^{\prime}, r^{\prime}$ and $g^{\prime}$ file contains about 300000 objects, $\sim 230000$ of them with flag $=0$. We shall employ and analyse this subset. The number of stars, having a photometric

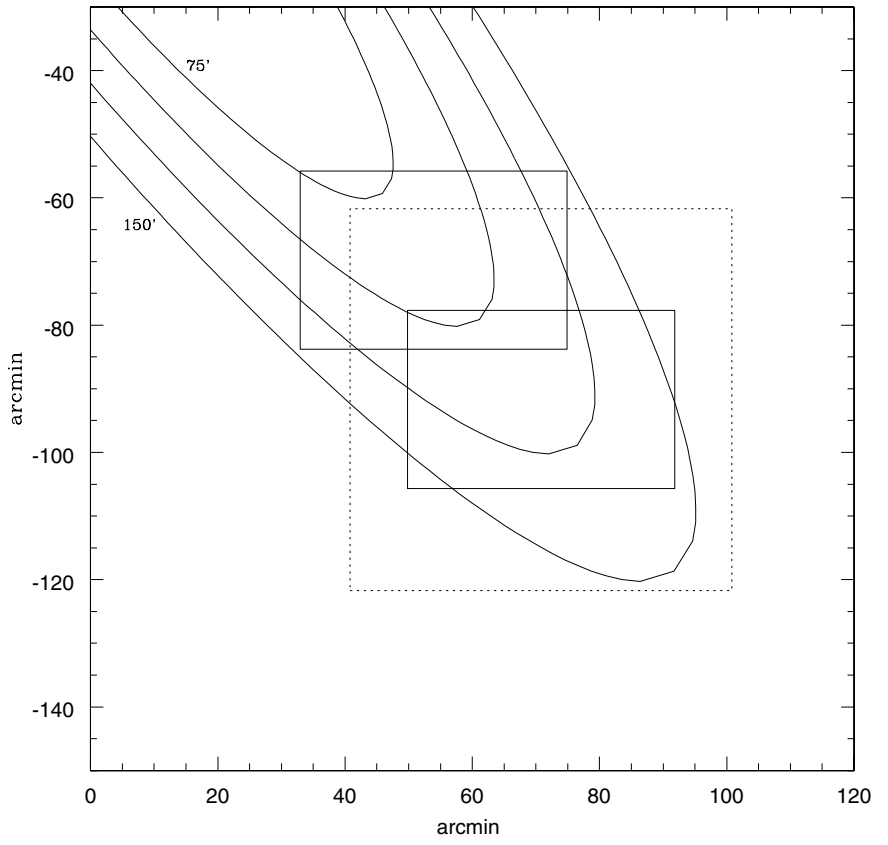

Fig. 1. Schematic representation of the south west disk of M31. Dotted square represents the Megacam field under discussion. Solid rectangles correspond to the SW1 and SW2 fields from Battinelli et al. (2003). The zero point of the coordinates coincides with the center of M31.

error smaller than a given value, varies from one file to the next. For example, the $i^{\prime}, r^{\prime}$ and $g^{\prime}$ files contain respectively 83000 , 121000 and 110000 stars with err $<0.10$ mag. However, when $i^{\prime}$ and $r^{\prime}$ are combined, the number of stars with colour error, $\sigma_{r i}<0.10$, is 50500 while for $g^{\prime}$ and $r^{\prime}$, the number of stars with $\sigma_{g r}<0.10$ is 56700 . When the three files are combined and, following our standard criterion only stars with $\sigma_{\text {irg }}=\left(\sigma_{r i}^{2}+\sigma_{g r}^{2}\right)^{1 / 2}<0.125$ are retained, the number of stars drops to 37000 . We shall see later that this small number is due to the presence of numerous faint red stars not well observed in the $g^{\prime}$ and $r^{\prime}$ filters. Finally the remaining $\sim 65000$ stars with flags $=1,2$, or 3 and corresponding to objects affected by close neighbours or/and originally blended will not be used for the magnitude and colour comparisons but will be needed later to cross-identify known C stars.

\subsection{The colour-magnitude diagrams}

A one square degree field in the direction of M31 must obviously include stars of different population. The major axis of its disk runs roughly diagonally across the Megacam field, from $18 \mathrm{kpc}$ to $33 \mathrm{kpc}$. At such distances the bulge population is completely negligible (Windrow et al. 2003), thus we see disk and halo stars of M31, our Galactic contributions (halo and disk stars) and numerous unresolved galaxies. We present, in Fig. 2, the two colour-magnitude diagrams (CMD) corresponding to the whole Megacam field for stars with flag $=0$ and colour error $<0.10$. The broken lines represent the limiting magnitudes which correspond to the magnitudes where the luminosity function drops to $50 \%$ of its peak value. These are found to be: $i^{\prime}=22.5, r^{\prime}=22.9$ and $g^{\prime}=24.0$. 

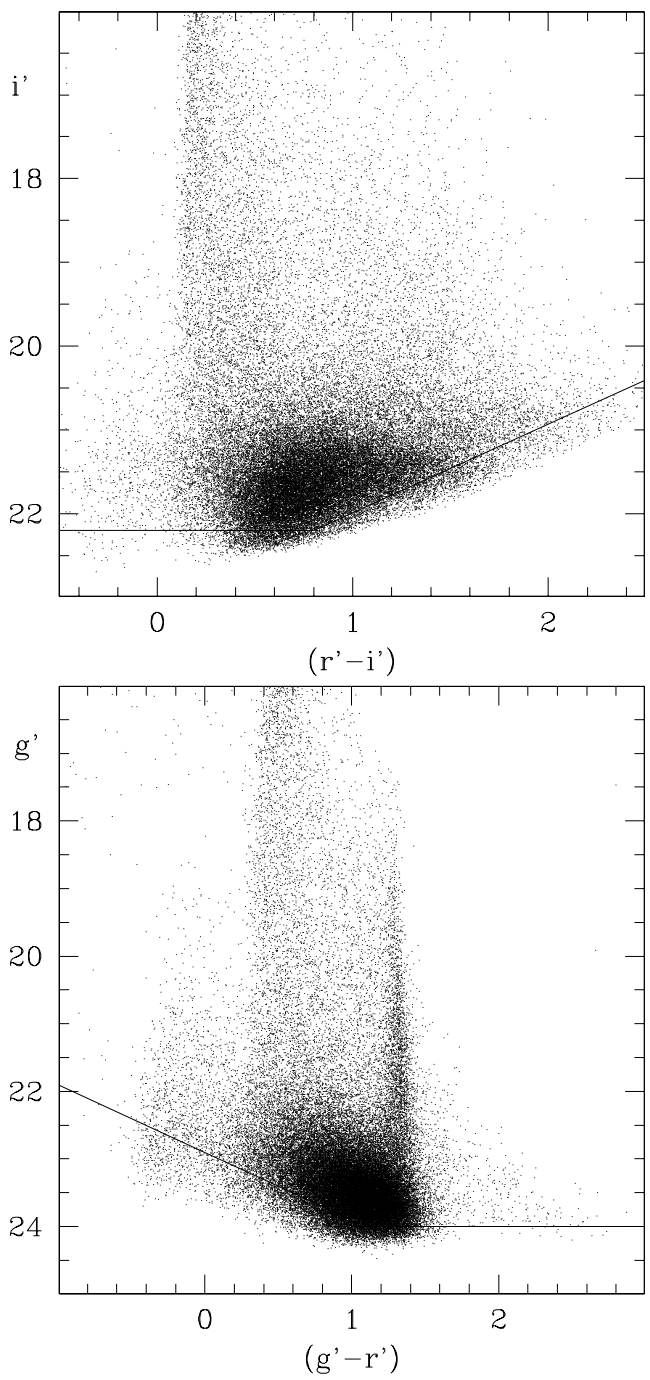

Fig. 2. Colour magnitude diagrams of the stars seen in the Megacam field. The lines correspond to the limiting magnitudes as defined in the text.

The top panel shows the $i^{\prime}$ magnitudes versus the $\left(r^{\prime}-\right.$ $\left.i^{\prime}\right)$ colours. Two features are conspicuous: the bright end of the red giant branch of M31, starting at $i^{\prime} \sim 21$ and extending far to the red; and the vertical ridge at $\left(r^{\prime}-i^{\prime}\right) \sim 0.10$. This ridge corresponds to Galactic G dwarfs, at the MS turnoff, seen along the line of sight. The colour location of this ridge is indicative of the reddening. According to Schlegel et al. (1998) the Galactic contribution to the reddening in this direction amounts to $E(B-V)=0.06$, which translates to $E\left(r^{\prime}-i^{\prime}\right)=0.04$.

The second CMD (lower panel of Fig. 2) has more interesting features. Blue main sequence stars are well separated from the bulk of M 31's stars, differential reddening within the star forming regions must be responsible for the diffuse appearance of the main sequence. The narrow vertical plume at $\left(g^{\prime}-r^{\prime}\right) \sim 1.4$ corresponds to stars of spectral type M, as the synthetic colours from Fukugita et al. (1996) demonstrate. This plume is also seen in the simulation of Galactic stellar objects by Fan (1999). The vertical ridge corresponding to Galactic $\mathrm{G}$ dwarf is also seen. Finally we see, in this CMD, a small population of stars extending at $\left(g^{\prime}-r^{\prime}\right)>1.7$. We identify these

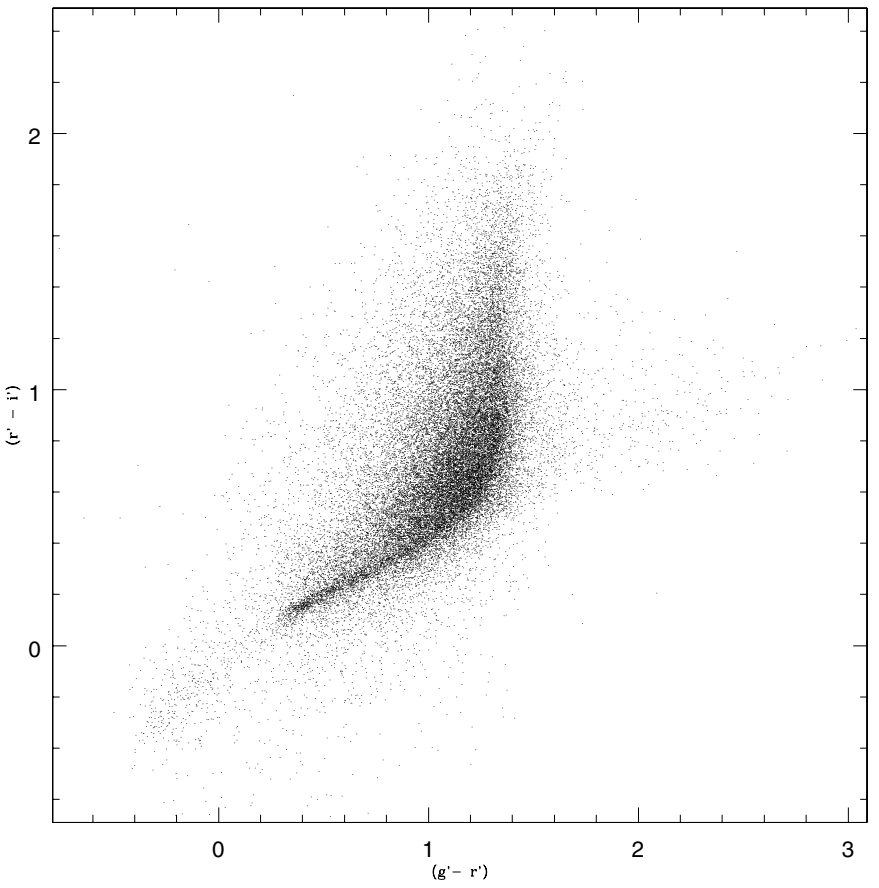

Fig. 3. Colour-colour diagram of the M 31 stars.

stars with extreme $\left(g^{\prime}-r^{\prime}\right)$ colours to $\mathrm{C}$ stars observed for the first time in the SLOAN colours by Krisciunas et al. (1998). This $\mathrm{C}$ star tail is seen to curve below the the limiting magnitude of the data, suggesting that some $\mathrm{C}$ stars must have been missed.

\subsection{The colour-colour diagram}

The colour-colour diagram of the Megacam field is displayed in Fig. 3. Again, only stars with flag $=0$ are included, we plot only stars fainter than $i^{\prime}=17.5$ to exclude some $(\sim 2000)$ of the foreground Galactic stars. $\mathrm{C}$ stars are seen to the right of the diagram, with colours reaching $\left(g^{\prime}-r^{\prime}\right)=3$.

We note, however, a number of points with extreme negative $\left(r^{\prime}-i^{\prime}\right)$ colours, seen at the bottom of the diagram. Investigation of their spatial distribution reveals that they are nearly all on the West side of the North-South CCD borders. The spurious $r^{\prime}$ magnitudes close to the borders are due to a $\sim 80$ pixel shift of one of the exposures relative to the two other ones. Thus, the instrumental magnitudes of about 200 stars near the CCD borders are wrong because they are not based on the right number of exposures. It is rather difficult to delete these stars from the database because we have lost their original $x, y$ CCD coordinates since we are now using equatorial coordinates.

\subsection{Cross identification of $M 31$ stars}

The goal of our study is to obtain the SDSS magnitude and colours of the known $\mathrm{C}$ stars in the disk of M31 which were identified from their position in the $(R-I)$ vs. $(\mathrm{CN}-\mathrm{TiO})$ plane. The $(\mathrm{CN}-\mathrm{TiO})$ technique is designed to identify cool N-type $\mathrm{C}$ stars. In the $(R-I)$ vs. $(\mathrm{CN}-\mathrm{TiO})$ plane, the warmer and 
fainter $\mathrm{C}$ stars are mixed with the late $\mathrm{K}$ or early $\mathrm{M}$ stars. For this reason the differentiation of $\mathrm{C}$ stars and $\mathrm{M}$ stars is limited to stars with $(R-I)_{0}>0.90$. Therefore, our approach will be somewhat different from the one employed by Margon et al. (2002) in their identification of faint high-latitude carbon stars (FHLCs) because we intend to deal exclusively with cool red C stars.

The first step in the cross identification is to match all stars with identical equatorial coordinates. To do so, we select two datasets: the CFH12K SW1 field consisting of 62007 stars for which we have $I,(R-I)$ and $(\mathrm{CN}-\mathrm{TiO})$ and a second set consisting of the 37000 stars in the Megacam field for which we have $i^{\prime},\left(r^{\prime}-i^{\prime}\right)$ and $\left(g^{\prime}-r^{\prime}\right)$. After a few iterations to minimize the $\Delta \alpha$ and $\Delta \delta$ we retain some 8000 pair of stars matched within 0.8 arcsec. The reason why such relatively small number of matches is obtained can be seen from Fig. 1, only $63 \%$ of SW1 overlaps with $20 \%$ of the Megacam field. In the region common to both fields there are $\sim 10000$ stars from Megacam and 35000 in the SW1 field.

We compare, in Fig. 4, the magnitudes and colours of these matched stars. The $i^{\prime}$ magnitudes are fainter than the $I$ magnitudes by $\sim 0.25 \mathrm{mag}$. The downward bulge, seen at the faint magnitude end, is simply due to the natural increase of the scatter for the faintest magnitudes. The asymmetry of the dispersion is explained by the missing stars with faint $i^{\prime}$ magnitudes. These stars are present in the $i^{\prime}$ file but, since they don't have matches in the $r^{\prime}$ or $g^{\prime}$ files, disappear from the $i^{\prime} r^{\prime} g^{\prime}$ Megacam dataset.

The relationship between the magnitudes can be expressed in the following ways, obtained from linear regressions:

$i^{\prime}=I+(0.289 \pm 0.010)+(0.1404 \pm 0.0078) \times(R-I)$

or

$I=i^{\prime}-(0.291 \pm 0.008)-(0.1717 \pm 0.0081) \times\left(r^{\prime}-i^{\prime}\right)$.

The comparison of the colours shows a significant scatter at the red end. This is simply a consequence of the rather bright $r^{\prime}$ limiting magnitude of our observations. The faint red stars have a lower photometry quality. The colour relationship is obtained by using only 900 stars with $i^{\prime}<20.5$. These stars have $\left(r^{\prime}-i^{\prime}\right)<2.0$.

$\left(r^{\prime}-i^{\prime}\right)=-(0.129 \pm 0.017)+(0.857 \pm 0.013) \times(R-I)$,

this is to be compared to the synthetic colours relation calculated by Fukugita et al. (1996). They quote $\left(r^{\prime}-i^{\prime}\right)=0.98(R-$ $I)-0.23$; for $(R-I)<1.15$ and $\left(r^{\prime}-i^{\prime}\right)=1.40(R-I)-0.72$; for $(R-I)>1.15$. We do not see in our data a break in the colour relation up to $\left(r^{\prime}-i^{\prime}\right) \approx 2.0$, where the brighter stars can be seen. For comparison, the Fukugita et al. relation is drawn in Fig. 4.

\subsection{Cross identification of $C$ stars}

Of the nearly one thousand M $31 \mathrm{C}$ stars identified by Battinelli et al. (2003) in fields SW1 and SW2, only 644 are in the Megacam field. Cross identification, with the same criterion described above, yields barely 129 matches, just $20 \%$ of the
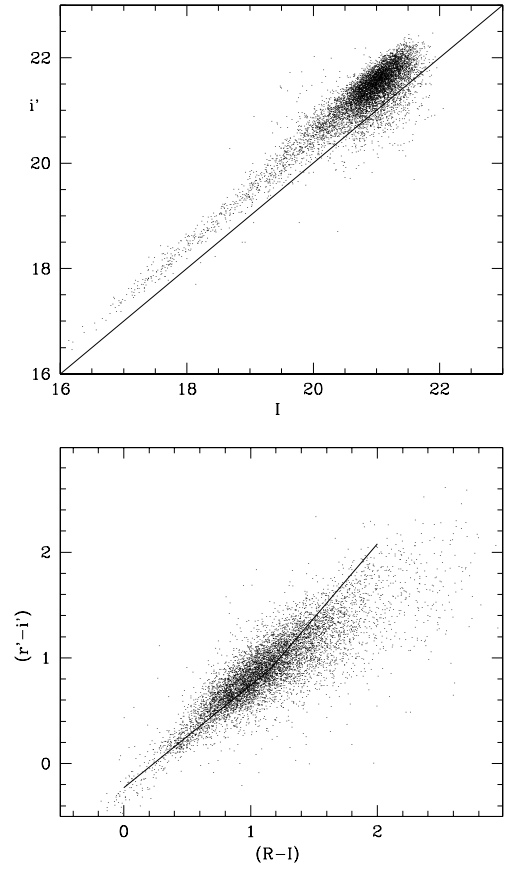

Fig. 4. Comparison of the magnitudes and colours of the 8000 stars matched between the two datasets. The top panel shows that the $i^{\prime}$ magnitudes are fainter than the $I$ magnitudes, the identity line is drawn. For the bottom panel, the lines represent the Fukugita et al. (1996) colour relation.

$\mathrm{C}$ stars in the field. There are two major reasons for this low success rate. The disk of M 31 shows a gradient of stellar surface density. The northern part of the Megacam field being more densely populated contains more $\mathrm{C}$ stars that are in a somewhat crowded environment. Many northern $\mathrm{C}$ stars were not matched. If we do not take into account the flag assigned by SExtrator to each star, and accept all stars irrespective of their flag, the number of carbon star matched increases to 245 . This is done without relaxing the photometric error criterion. The second explanation for the low success rate comes from the fact that the limiting $r^{\prime}$ and $g^{\prime}$ magnitudes are not as faint as the $\mathrm{CFH} 12 \mathrm{~K}$ data, thus the redder $\mathrm{C}$ stars are missed. Figure 5 compares the $(R-I)_{0}$ colour distribution of the known $\mathrm{C}$ stars with those cross identified in the $i^{\prime}$ and $g^{\prime}$ files. Obviously the red stars are missing in the $g^{\prime}$ file and also, to a lesser extent in the $r^{\prime}$ file, not shown here.

The magnitude and colour distributions of the known $\mathrm{C}$ stars and those identified in our Megacam data are shown in Fig. 6. The top panel indicates the number of $\mathrm{C}$ stars cross identified depends moderately on the apparent $i^{\prime}$ magnitude. This implies that the crowding is the most important factor. The lower panel confirms that red stars are missing from our Megacam sample.

Figure 7 presents the SDSS colour-colour diagram of the 245 C stars recovered in our Megacam data, when no flag restriction is applied. The three solid lines outline the acceptance limits adopted by Margon et al. (2002) for the FHLCs. As expected, our $\mathrm{C}$ stars are redder than these limits in $\left(r^{\prime}-i^{\prime}\right)$ as well as in $\left(g^{\prime}-r^{\prime}\right)$. Since our earlier adopted limit of $(R-I)_{0}=0.90$ corresponds to $\left(r^{\prime}-i^{\prime}\right)=0.64$, very few if any $\mathrm{C}$ stars should 


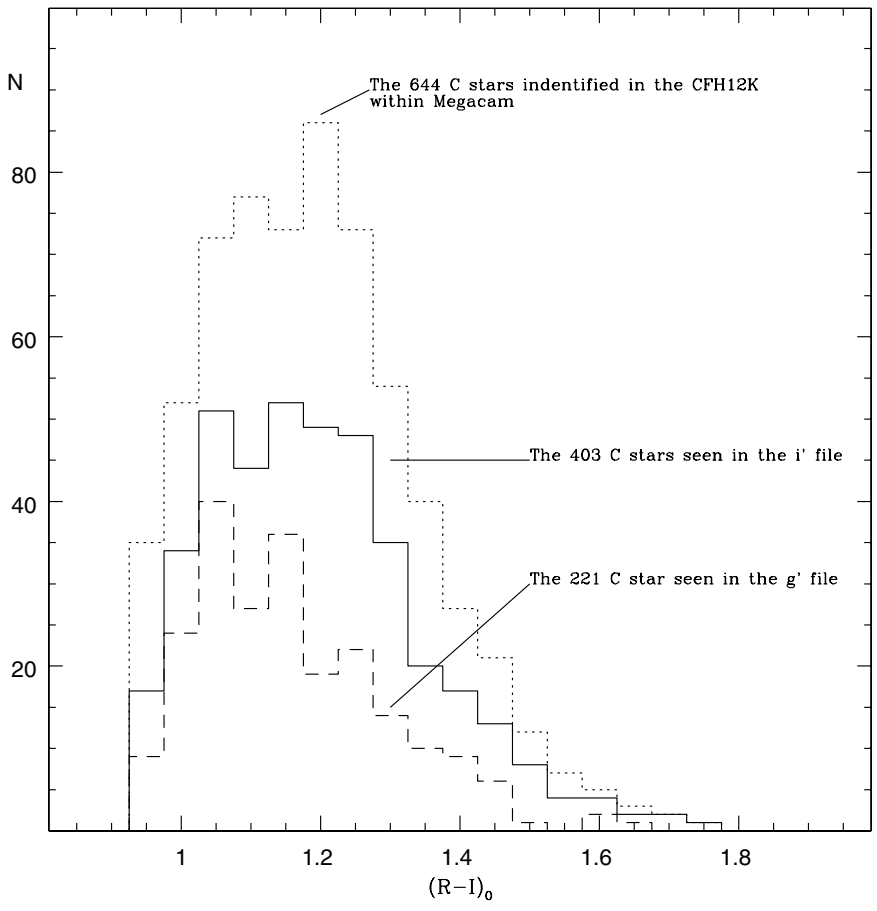

Fig. 5. Colour distributions of the $\mathrm{C}$ stars and those cross-identified with the Megacam data.
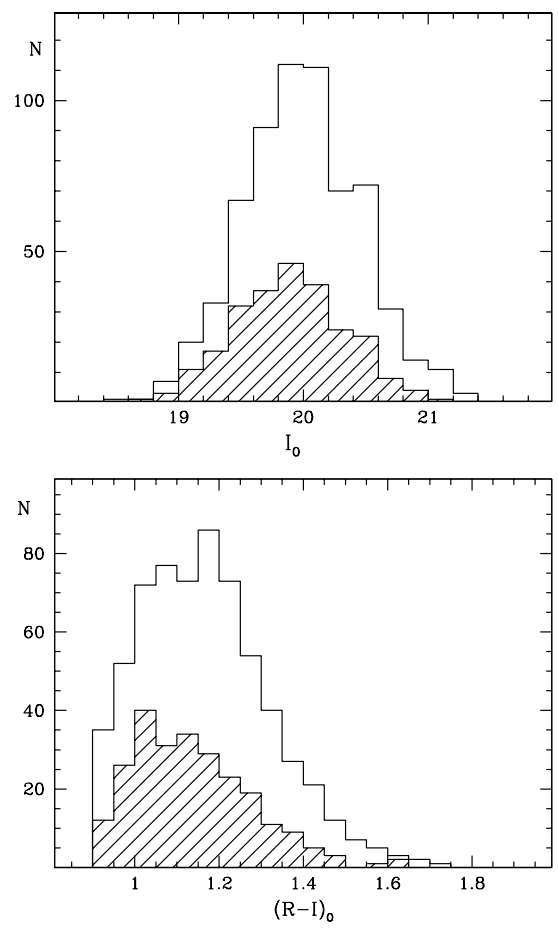

Fig. 6. Magnitude and colour distributions of the 644 known C stars are compared to the distribution of the $245 \mathrm{C}$ stars cross-identified with our three magnitude Megacam file (shaded histograms).

have bluer $\left(r^{\prime}-i^{\prime}\right)$ colours. These Megacam observations, sampling the disk of M 31 where numerous $\mathrm{K}$ giants are present, are certainly not ideal to identify bluer $\mathrm{C}$ stars which can easily be confused with the bulk of the $\mathrm{K}$ giants. We draw, somewhat arbitrarily, the dashed lines corresponding to our adopted colour limits for the M31 cool C star population. The extreme

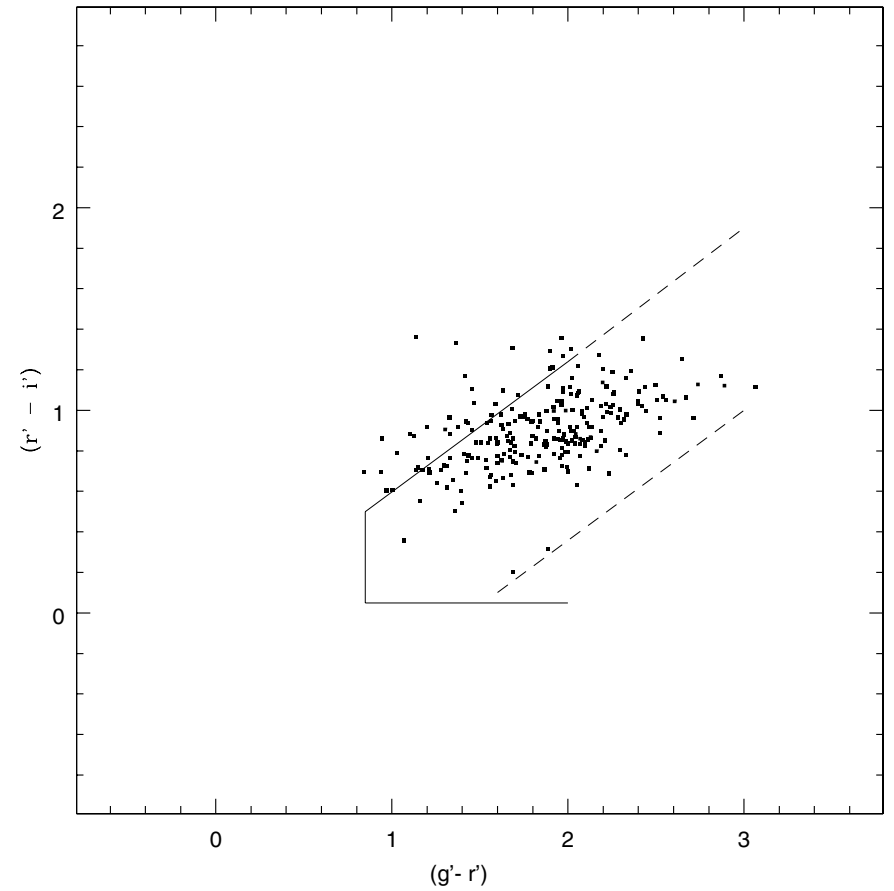

Fig. 7. Colour-colour diagram of the $245 \mathrm{C}$ stars, identified from their $(\mathrm{CN}-\mathrm{TiO})$ index. The three solid lines define the $\mathrm{C}$ star acceptance limits adopted by Margon et al. (2002). The dashed lines trace our adopted upper and lower limits.

upper and lower points correspond to stars that have calculated magnitude and colour (given by Eqs. (2) and (3)) which differ substantially from the $\mathrm{CFH} 12 \mathrm{~K}$ values. The variability of $\mathrm{C}$ stars could be responsible or, more likely, spurious matches are always possible in a crowded field. The $129 \mathrm{C}$ stars matched to the flag $=0$ data are indistinguishable in the colour-colour plane.

\subsection{Selection criteria for cool C stars}

A close-up of Fig. 3, along with our adopt boundaries for $\mathrm{C}$ stars is displayed in Fig. 8. It is obvious that the blue limit adopted by Margon et al. (2002) is of little use to us. Indeed, the numerous $\mathrm{K}$ stars seen in the M 31 disk, overwhelm the few $\mathrm{C}$ stars with $\left(g^{\prime}-r^{\prime}\right) \approx 1.5$. This, to a so large extent, that to exclude as much as possible $\mathrm{K}$ and $\mathrm{M}$ stars it would seem necessary to adopt a conservative blue limit around $\left(g^{\prime}-r^{\prime}\right) \approx 1.7$. We describe how we can better determine this colour limit.

For the 8000 stars cross-identified we have their $(R-I)$, $(\mathrm{CN}-\mathrm{TiO}),\left(g^{\prime}-r^{\prime}\right)$ and $\left(r^{\prime}-i^{\prime}\right)$. We can then identify the $\mathrm{C}$ and $\mathrm{M}$ stars by applying our criteria based on $(R-I)$ and $(\mathrm{CN}-\mathrm{TiO})$ colours. Thus we can calculate the number of $\mathrm{C}$ or $\mathrm{M}$ stars having a $\left(g^{\prime}-r^{\prime}\right)$ larger than a certain limit. The numbers of $\mathrm{C}$ stars $\left(N_{\mathrm{C}}\right)$ and $\mathrm{M}$ stars $\left(N_{\mathrm{M}}\right)$ selected for different $\left(g^{\prime}-r^{\prime}\right)$ lower limits are displayed in Fig. 9. As we shift the blue limit to redder colours the number of $\mathrm{M}$ stars drops appreciably while the number of $\mathrm{C}$ stars decreases slightly. Figure 10 presents the variation of the ratio of the number of $\mathrm{C}$ stars to the sum $\left(N_{\mathrm{C}}+N_{\mathrm{M}}\right)$ for various $\left(g^{\prime}-r^{\prime}\right)$ limits. The dashed curve is the ratio of $N_{\mathrm{C}}$ relative to the original $245 \mathrm{C}$ stars. 


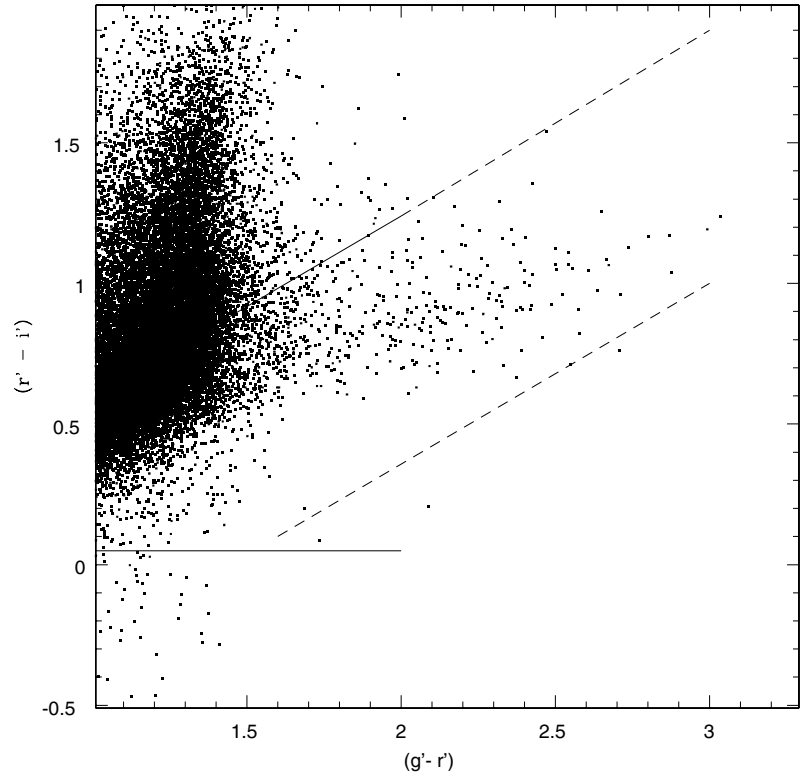

Fig. 8. Close-up of the colour-colour diagram for stars with flag $=0$. The dashed lines represent the adopted boundaries.

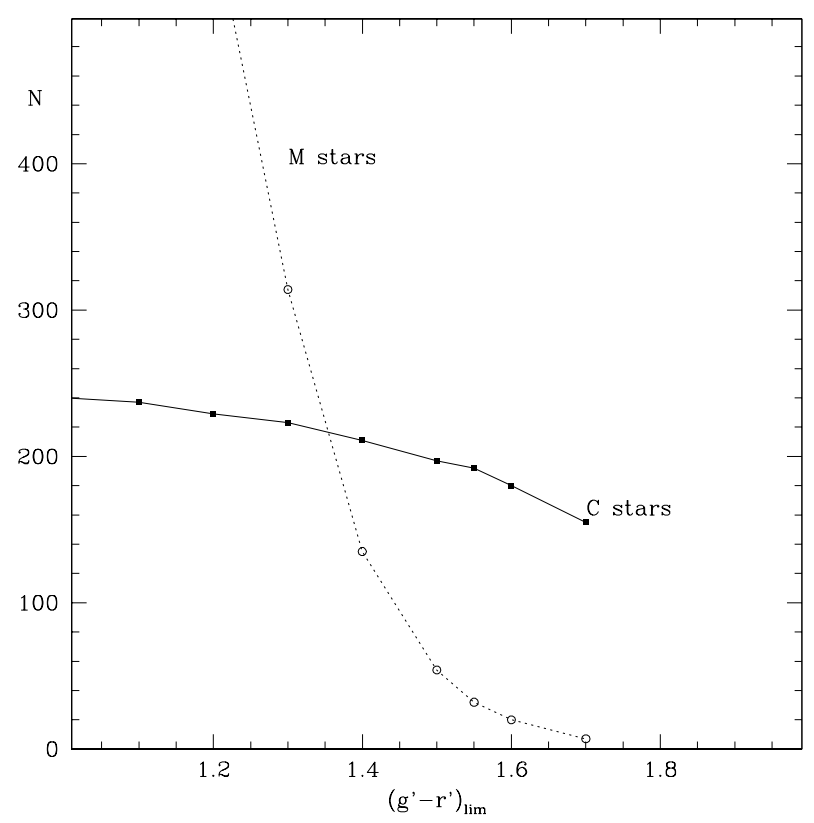

Fig. 9. The numbers of $\mathrm{C}$ and $\mathrm{M}$ stars retrieved from samples having different colour limits show that the selection of $\mathrm{M}$ stars is very sensitive to the colour limit.

We conclude, from this figure, that a reasonable colour limit is $\left(g^{\prime}-r^{\prime}\right)=1.55$. At this limit $86 \%$ of the stars are $\mathrm{C}$ stars and we lose $20 \%$ of the $\mathrm{C}$ stars which have bluer colours. Furthermore, as we have previously explained, the $\mathrm{C}$ stars detected by this technique represent only a fraction of the total cool C star population because our Megacam observations do not have sufficiently deep exposures.

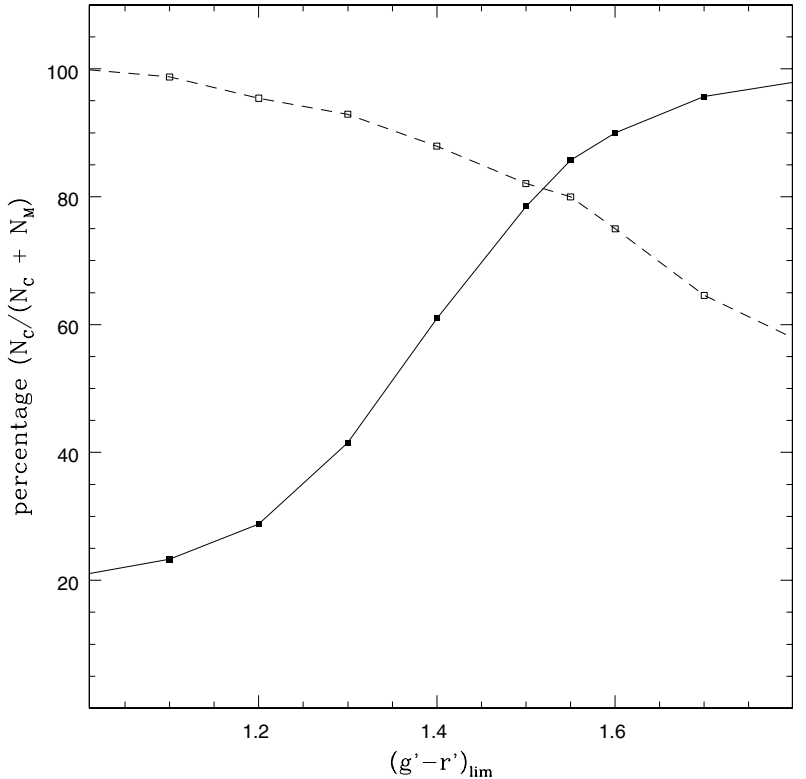

Fig. 10. The change of the ratio $\left(N_{\mathrm{C}} /\left(N_{\mathrm{C}}+N_{\mathrm{M}}\right)\right.$ for different colour limits. The dashed curve is the percentage of the $245 \mathrm{C}$ star retained.

\section{Discussion}

\subsection{Properties of $C$ stars}

Applying the above colour limits to our Megacam data file, for stars with flag $=0$ and colour $\sigma_{i r g}<0.125$, yields a sample of $480 \mathrm{C}$ stars candidates. According to Fig. 9, there should be some $70 \mathrm{~K}$ or $\mathrm{M}$ stars polluting this sample. Cross-identification with the known C stars in M 31 results in 102 matches. However, a cross-identification with the whole CFH12K SW1 database reveals that 17 stars are in fact M stars. These few stars are thus deleted from our sample. The equatorial coordinates, given in degrees, the magnitude, the colours and their attached errors as determined by SExtractor of these remaining 463 stars are listed in Table 2 . The $\sigma_{g r}$ being twice as large than the $\sigma_{r i}$ is explained by the faintness of $\mathrm{C}$ stars in the $g^{\prime}$ band. 361 of them are newly identified C stars located almost all at larger radial distances than the ones previously known.

The $i^{\prime}$ luminosity function of the $463 \mathrm{C}$ stars candidates is displayed in Fig. 11. A Gaussian is fitted by eyes over the distribution. Their mean apparent magnitude $\left\langle i^{\prime}\right\rangle=20.64$, with a variance of 0.31 , their mean colours are: $\left\langle\left(r^{\prime}-i^{\prime}\right)\right\rangle=0.86$ and $\left\langle\left(g^{\prime}-r^{\prime}\right)\right\rangle=1.86$. Using Eq. (2), this mean magnitude corresponds to $\langle I\rangle=20.20$, a value to be compared with $\left\langle I_{0}\right\rangle=19.94$, obtained for the M31 C star population by Battinelli et al. (2003). Taking into account a mean extinction, of the order of $A_{I}=0.12$, implies that we have acquired essentially the same stellar population even though using different colour criteria.

\subsection{The M31 disk and its intermediate-age population}

C stars can be used to map the spatial distribution of the intermediate-age population. To do so, we calculate the surface density per $\operatorname{arcmin}^{2}$ in elliptical annuli of $10^{\prime}$ width having the 
Table 2. Complete Table 2 is available in electronic form at the CDS. A portion is shown here for guidance regarding its form and content. Units of right ascensions and declination (J2000) are in degrees.

\begin{tabular}{ccccccccc}
\hline \hline id & RA & Dec & $i^{\prime}$ & $\sigma_{r}$ & $\left(r^{\prime}-i^{\prime}\right)$ & $\sigma_{r-i}$ & $\left(g^{\prime}-r^{\prime}\right)$ & $\sigma_{g-r}$ \\
\hline 1 & 8.6219311 & 39.6322746 & 20.877 & 0.030 & 0.994 & 0.043 & 2.415 & 0.113 \\
2 & 8.6224728 & 39.7282753 & 20.107 & 0.018 & 0.850 & 0.025 & 1.791 & 0.042 \\
3 & 8.6282034 & 39.8302460 & 20.585 & 0.026 & 1.134 & 0.040 & 2.209 & 0.097 \\
4 & 8.6309576 & 39.3995628 & 20.726 & 0.028 & 0.471 & 0.034 & 1.571 & 0.042 \\
5 & 8.6383266 & 39.3832932 & 20.548 & 0.036 & 0.899 & 0.048 & 1.721 & 0.073 \\
6 & 8.6383543 & 39.8645439 & 21.190 & 0.036 & 0.950 & 0.051 & 2.027 & 0.107 \\
7 & 8.6440039 & 39.7481689 & 21.465 & 0.043 & 0.610 & 0.054 & 2.024 & 0.093 \\
8 & 8.6491823 & 39.6935310 & 20.527 & 0.022 & 0.974 & 0.032 & 2.636 & 0.097 \\
9 & 8.6501064 & 40.1469650 & 19.649 & 0.018 & 0.793 & 0.023 & 1.691 & 0.035 \\
10 & 8.6624346 & 39.3817787 & 20.508 & 0.021 & 1.049 & 0.033 & 2.192 & 0.075 \\
11 & 8.6695499 & 39.4705505 & 20.596 & 0.023 & 0.917 & 0.032 & 2.112 & 0.067 \\
12 & 8.6699352 & 39.5931396 & 20.262 & 0.020 & 0.844 & 0.028 & 1.918 & 0.051 \\
\hline
\end{tabular}

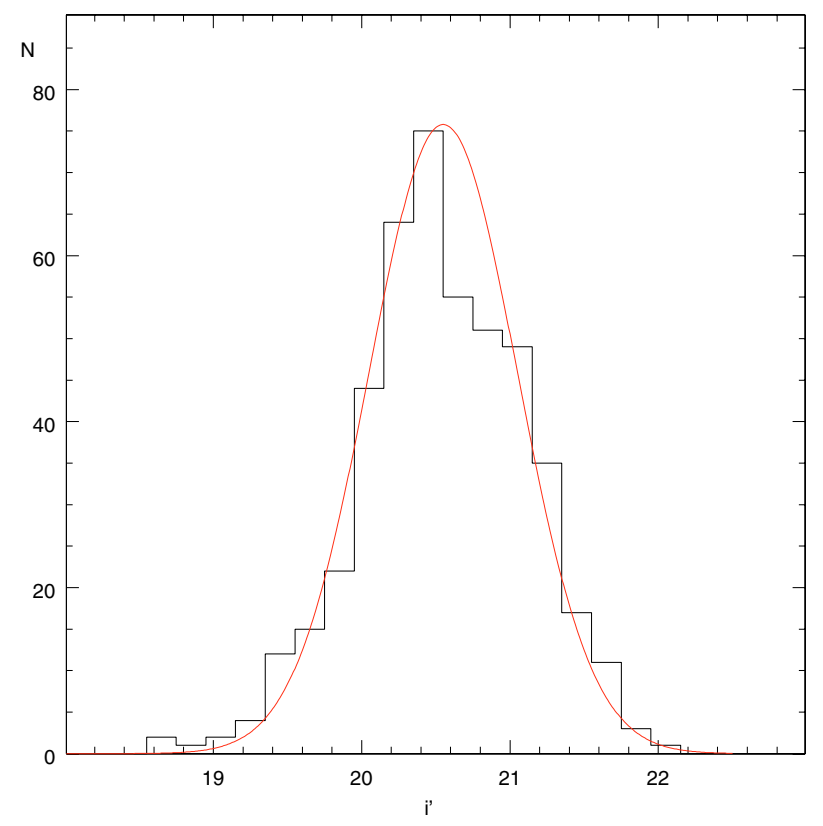

Fig. 11. Luminosity function of the $463 \mathrm{C}$ stars identified by applying our colour criterion.

shape of the M 31 apparent disk. Such ellipses are sketched in Fig. 1. We adopt for the disk of M31 a position angle of the major axis of $37.7^{\circ}$ and an ellipticity $\epsilon=0.787$. Because of the huge size of M31, even compared to our one $\mathrm{deg}^{2}$ field, we are observing only sectors of annuli of quite different angular length. Even though the radial distance along the major axis reaches only $\sim 150^{\prime}$, we can sample larger radial distances on the edge of the field away from the major axis. The top panel of Fig. 12 presents the $\mathrm{C}$ star surface density as a function of the radial angular distances. The bottom panel shows the surface density of the all stars detected by SExtractor fainter than $i^{\prime}=17.5$ to exclude the brighter Galactic stars. These stars are mostly M31's red giants. Their surface density reaches a plateau at $\sim 170^{\prime}(\sim 35 \mathrm{kpc})$ this position should correspond to the edge of the stellar disk. If we adopt, by averaging the last 7 points, 36.54 stars/ $\operatorname{arcmin}^{2}$ for the density outside of the disk, the declining slope of the surface density corresponds to a scale length of $4.9 \pm 0.4 \mathrm{kpc}$. This is in excellent agreement with the scale length of C stars determined by Battinelli et al. (2003) and the one derived by Walterbos \& Kennicutt (1988) from multicolour integrated surface photometry.

The density profile of $\mathrm{C}$ stars is much more irregular. The flattening of the profile at short distances is most probably due to the fact that SExtractor has difficulties dealing with crowded fields. For $\mathrm{C}$ stars only stars with $\sigma_{i r g}<0.125$ are selected while stars of all errors are included in the bottom panel. Contrary to the bulk of stars, C stars are seen up to $\sim 190^{\prime}$, $(\sim 40 \mathrm{kpc})$ where a sharp drop is observed. The last three points represent only five C stars farther than $200^{\prime}$. This confirms the identification of $\mathrm{C}$ stars along the major axis of the disk by Battinelli \& Demers (2005) who found one C star at $40 \mathrm{kpc}$, well outside of the Megacam field.

The fact that $\mathrm{C}$ stars are seen beyond "the edge of the disk" suggests that a tenuous thick disk, containing intermediate-age stars, must be present beyond the detectable edge. It density contrast, relative to the halo population, may be too low for easy detection. Since C stars are seen behind a zero foreground they can be seen even in extremely low density environment.

\subsection{The $C / M$ ratio}

We have confirmed that the SDSS colours are useful to identify cool C stars. The next step is to adopt a colour criterion applied to the $\mathrm{M}$ stars, thus allowing the determination of the $\mathrm{C} / \mathrm{M}$ ratio. According the Hawley et al. (2002), M0 dwarfs have $\left\langle\left(r^{\prime}-i^{\prime}\right)\right\rangle=0.91 \pm 0.24$. Unfortunately, because of the width and upper colour limit of the $\mathrm{C}$ star zone in the colour-colour 

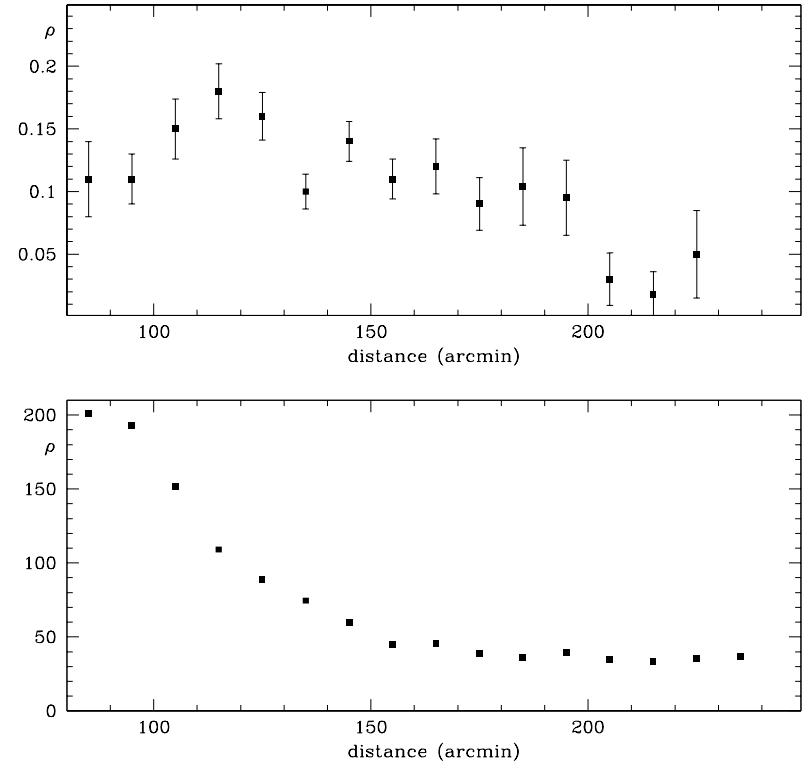

Fig. 12. The surface densities, stars per $\operatorname{arcmin}^{2}$ for $\mathrm{C}$ stars (top) and all stars with $i^{\prime}>17.5$ (bottom).

diagram it appears quite difficult to discriminate $\mathrm{M}$ stars from C stars near such $\left(r^{\prime}-i^{\prime}\right)$ colour. However, M3 stars have $\left\langle\left(r^{\prime}-i^{\prime}\right)\right\rangle=1.29 \pm 0.32$ a colour that cannot be confused with C stars. Therefore, we adopt for colour limits of the M 3+ stars: $\left(r^{\prime}-i^{\prime}\right)=1.3$ and $\left(g^{\prime}-r^{\prime}\right)=1.5$, to separate them slightly from $C$ stars. We have $\sim 6000$ such stars (with $\sigma_{i r}<0.10$ ) in our database. The limiting magnitude of this sample, because of the redness of the stars, reaches $i^{\prime} \approx 22$, which translates into $I=21.5$.

M3+ counts, in the same $10^{\prime}$ wide elliptical annuli previously adopted, show a plateau for distances larger than $170^{\prime}$. We average these outer counts to obtain an estimate of the foreground/background density. In our case it corresponds to $1.06 \pm 0.03 \mathrm{M} 3+$ stars per $\operatorname{arcmin}^{2}$. A value we substract from the observed M3+ density. This density is to be compared to the M3+ density of 0.8 stars/ $\operatorname{arcmin}^{2}$ we calculate from Durrell's et al. (2001) observations of a remote field near M 31 .

Figure 13 presents the $\mathrm{C} / \mathrm{M} 3+$ ratio as a function of galactocentric distances. As expected, for a decrease of metallicity with radial distances, the number of $\mathrm{C}$ stars increases relative to the number of M3+ stars. Such behavior, for example, has been observed in M 33 by Rowe et al. (2004).

\subsection{Colour and magnitude trend with the galactocentric distance}

Our previous two investigations of the outer disk of M 31 have revealed the existence of a mild decrease of the $I$ luminosity of C stars with increasing galactocentric distances (see Battinelli $\&$ Demers 2005). It is therefore worth to inspect the behaviour of the newly identified $\mathrm{C}$ stars which extend the previous surveys up to nearly $240^{\prime}$. Figure 14 shows the magnitude and colour as a function of the galactocentric distance for the newly identified $\mathrm{C}$ stars along with the nearly 1000 previously know (Battinelli et al. 2003; Battinelli \& Demers 2005). Megacam $i^{\prime}$

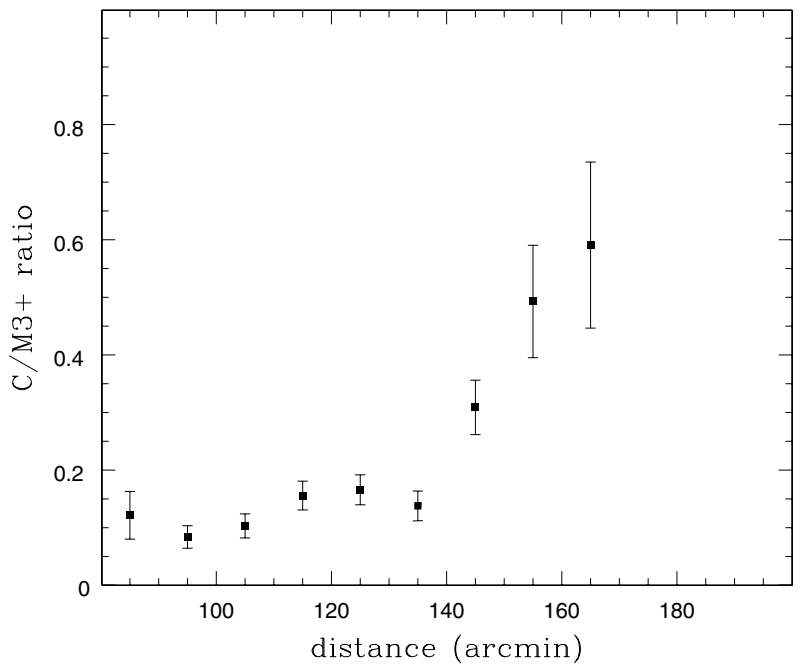

Fig. 13. The $\mathrm{C} / \mathrm{M} 3+$ ratio determined for elliptical annuli of the shape of M31's disk.
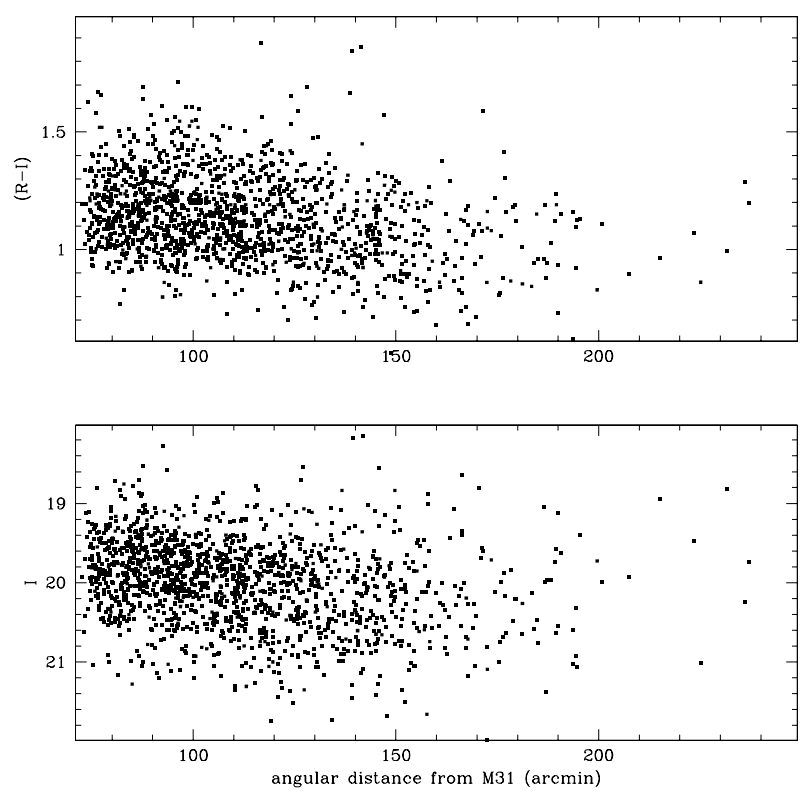

Fig. 14. Colour and magnitude of $\mathrm{C}$ stars as a function of the galactocentric distances.

were converted into Kron-Cousins $I$ using Eq. (2). A certain radial fading of the luminosity and a gradual disappearance of the reddest $C$ stars is evident. In principle, the luminosity fading may be explained by an increase of the metallicity at large galactocentric distances. Indeed, it is well know that the higher the metallicity the lower is the $\mathrm{C}$ star luminosity. This explanation is however unsatisfactory since both the observed colour gradient, shown in the top panel of Fig. 14, and the C/M behaviour in Fig. 13 point definitely towards a metallicity decrease in the outer part of the Andromeda disk. Age could, however, be responsible for the observed luminosity fading. Indeed, theoretical models for simple stellar populations of intermediate age (see e.g. Fig. 11 in Marigo et al. 1999) suggest that the brightest $\mathrm{C}$ stars disappear when the age of the population increases. A radial increase of the age of the youngest C stars (or equivalently a decrease of the mass of the most 
massive C stars) can overwhelm the metallicity effect and explain the luminosity trend. It is clear that, beyond this qualitative considerations, an answer to the question is possible only through a full modeling - which could also account for the observed metallicity and density radial trends - of the composite stellar population in the outer disk.

\section{Conclusion}

The SDSS filters offer an alternative method to identify C stars and also late $\mathrm{M}$ stars. Contrary to the $(\mathrm{CN}-\mathrm{TiO})$ technique, it is difficult with the SDSS filters to isolate C stars from M 0 stars. Furthermore, because numerous $\mathrm{C}$ stars have quite large $\left(g^{\prime}-r^{\prime}\right)$ colour the exposure time to reach the desired $g^{\prime}$ magnitude can be very long relative to the $i^{\prime}$ exposure. In term of telescope time both techniques require approximately the same total exposures since the $\mathrm{CN}$ and $\mathrm{TiO}$ exposures must be at least three to four times the $I$ exposures.

The SDSS approach provides, however, uncontested advantages for two aspects of the extragalactic $\mathrm{C}$ stars survey. These filters are available on the new generation of large mosaic detectors, such as Megacam. They allow the survey of an entire nearby galaxy in a relatively short time. We have recently followed this approach to survey $4 \mathrm{deg}^{2}$ around NGC 6822 where nearly $900 \mathrm{C}$ stars are already known in its extended halo (Letarte et al. 2002). Optical imagers, albeit of small field size, available on some large telescopes, such as Gemini, offer - sometimes exclusively - SDSS filters. Because of the lack of the general availability of the $\mathrm{CN}$ and $\mathrm{TiO}$ filter, the SDSS approach must be adopted for any attempt to survey $\mathrm{C}$ stars among the neighbours of the Local Group.
Acknowledgements. This research is funded in parts (S.D.) by the Natural Science and Engineering Council of Canada. We are grateful to Yannick Mellier and the Terapix team to have so promptly accepted to measure our Megacam data.

\section{References}

Albert, L., Demers, S., \& Kunkel, W. E. 2000, AJ, 119, 2780 Battinelli, P., \& Demers, S. 2005, A\&A, 430, 905

Battinelli, P., Demers, S., \& Letarte, B. 2003, AJ, 125, 1298

Bertin, E., \& Arnouts, S. 1996, A\&AS, 117, 393

Brewer, J. P., Richer, H. B., \& Crabtree, D. R. 1995, AJ, 109, 2480

Cioni, M.-R. L., \& Habing, H. J. 2005, A\&A, 429, 837

Demers, S., Dallaire, M., \& Battinelli, P. 2002, AJ, 123, 3428

Demers, S., Battinelli, P., \& Letarte, B. 2003, A\&A, 410, 795

Downes, R. A., Margon, B., Anderson, S., et al. 2004, AJ, 127, 2838

Durrell, P. R., Harris, W. E., \& Pritchet, C. J. 2001, AJ, 121, 2557

Fan, X. 1999, AJ, 117, 2528

Fukugita, M., Ichikawa, T., Gunn, J. E., et al. 1996, AJ, 111, 1748

Harbeck, D., Gallagher, J. S., \& Grebel, E. K. 2004, AJ, 127, 2711

Hawley, S. L., Covey, K. R., \& Knapp, G. R. 2002, AJ, 123, 3409

Krisciunas, K., Margon, B., \& Szkody, P. 1998, PASP, 110, 1342

Letarte, B., Demers, S., Battinelli, P., \& Kunkel, W. E. 2002, AJ, 123, 832

Marigo, P., Girardi, L., \& Bressan, A. 1999, A\&A, 344, 123

Margon, B., Anderson, S. F., Harris, H. C., et al. 2002, AJ, 124, 1651

Nowotny, W., Kerschbaum, F., Olofsson, H., \& Schwarz, H. E. 2003, A\&A, 403, 93

Rowe, J. F., Richer, H. B., Brewer, J. P., \& Crabtree, D. R. 2004, AJ, in press

Schlegel, D., Finkbeiner, D., \& Davis, M. 1998, ApJ, 500, 525

Smith, J. A., Tucker, D. L., Kent, S., et al. 2002, AJ, 123, 2121

Walterbos, R. A. M., \& Kennicutt, R. C. 1988, A\&A, 198, 61

Windrow, L. M., Perrett, K. M., \& Suyu, S. H. 2003, ApJ, 588, 311 